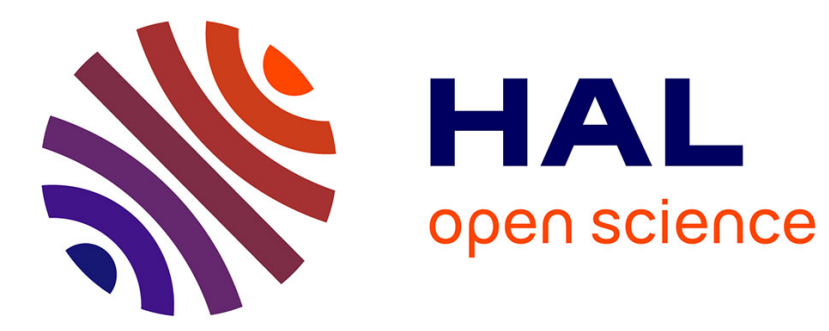

\title{
Estimation and segmentation in non-Gaussian POLSAR clutter by SIRV stochastic processes
}

Gabriel Vasile, Jean-Philippe Ovarlez, Frédéric Pascal

\section{To cite this version:}

Gabriel Vasile, Jean-Philippe Ovarlez, Frédéric Pascal. Estimation and segmentation in nonGaussian POLSAR clutter by SIRV stochastic processes. IGARSS 2009 - IEEE International Geoscience and Remote Sensing Symposium, Jul 2009, Le Cap, South Africa. pp.III-963-III-966, 10.1109/IGARSS.2009.5417935 . hal-02493508

\section{HAL Id: hal-02493508 https://hal.science/hal-02493508}

Submitted on 27 Feb 2020

HAL is a multi-disciplinary open access archive for the deposit and dissemination of scientific research documents, whether they are published or not. The documents may come from teaching and research institutions in France or abroad, or from public or private research centers.
L'archive ouverte pluridisciplinaire HAL, est destinée au dépôt et à la diffusion de documents scientifiques de niveau recherche, publiés ou non, émanant des établissements d'enseignement et de recherche français ou étrangers, des laboratoires publics ou privés. 


\title{
ESTIMATION AND SEGMENTATION IN NON-GAUSSIAN POLSAR CLUTTER BY SIRV STOCHASTIC PROCESSES
}

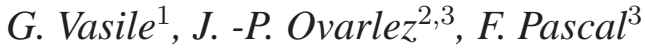 \\ ${ }^{1}$ : Grenoble-Image-sPeach-Signal-Automatics Lab, CNRS / Grenoble-INP, FRANCE, gabriel.vasile@gipsa-lab.grenoble-inp.fr \\ 2 : French Aerospace Lab, ONERA DEMR/TSI, FRANCE, ovarlez@onera.fr \\ ${ }^{3}$ : SONDRA, Supélec, FRANCE, frederic.pascal@supelec.fr
}

\begin{abstract}
In the context of non-Gaussian polarimetric clutter models, this paper presents an application of the recent advances in the field of Spherically Invariant Random Vectors (SIRV) modelling for coherency matrix estimation in heterogeneous clutter. The complete description of the POLSAR data set is achieved by estimating the span and the normalized coherency independently. The normalized coherency describes the polarimetric diversity, while the span indicates the total received power. Based on the SIRV model, a new maximum likelihood distance measure is introduced for unsupervised POLSAR segmentation. The proposed method is tested with airborne POLSAR images provided by the RAMSES system.
\end{abstract}

Index Terms- POLSAR, estimation, segmentation

\section{INTRODUCTION}

In a particular frequency band, the wave-media interactions over distributed areas are generally studied using the polarimetric covariance matrix (called also coherency when vectorizing in the Pauli basis). In general, POLSAR data are locally modelled by the multivariate, zero mean, circular Gaussian probability density function, which is completely determined by the covariance matrix.

The recently launched POLSAR systems are now capable of producing high quality images of the Earth's surface with meter resolution. The decrease of the resolution cell offers the opportunity to observe much thinner spatial features than the decametric resolution of the up-to-now available SAR images. Recent studies [1] show that the higher scene heterogeneity leads to non-Gaussian polarimetric clutter modelling, especially for urban areas.

This paper presented a new estimation scheme for deriving normalized coherency matrices and the resulting estimated span with high resolution POLSAR images. The proposed Fixed Point estimator is independent on the span PDF and represents an approximate ML estimator for a large class of stochastic processes obeying the SIRV model. For SIRV clutter, a new approximate ML distance measure is introduced for unsupervised POLSAR classification.

\section{CLUTTER MODELLING BY SIRV PROCESSES}

For Gaussian polarimetric clutter model, the estimation of the polarimetric coherency matrix is treated in the context of POLSAR speckle filtering. In the context of non-Gaussian polarimetric clutter models, several studies tackled POLSAR parameter estimation using the product model. Spherically Invariant Random Vectors (SIRV) and their applications to estimation and detection in communication theory were firstly introduced by Kung Yao [2]. In the context of POLSAR data, the clutter is modelled as SIRV, a non-homogeneous Gaussian process with random power: its randomness is induced by variations in the radar backscattering over different polarization channels. Consequently, the POLSAR target vector $\mathbf{k}$ is defined as the product between the independent complex Gaussian vector $\mathbf{z}$ with zero mean and covariance matrix $[M]=E\left\{\mathbf{z Z}^{\dagger}\right\}$ (representing the speckle) and the root of a positive random variable $\tau$ (representing the texture):

$$
\mathbf{k}=\sqrt{\tau} \mathbf{z},
$$

where $\nmid$ denotes the conjugate transpose operator and $E\{\ldots\}$ the mathematical expectation. In Eq. 11 the covariance matrix is an unknown parameter which can be estimated from Maximum Likelihood (ML) theory.

For a given $[M]$ the texture ML estimator is:

$$
\widehat{\tau}_{i}=\frac{\mathbf{k}_{i}^{\dagger}[M]^{-1} \mathbf{k}_{i}}{m} \text {. }
$$

The ML estimator of the normalized covariance matrix in the deterministic texture case is obtained by cancelling the gradient of the generalized likelihood with respect to $[M]$ as the solution of the following recursive equation:

$$
[\widehat{M}]_{F P}=f\left([\widehat{M}]_{F P}\right)=\frac{m}{N} \sum_{i=1}^{N} \frac{\mathbf{k}_{i} \mathbf{k}_{i}^{\dagger}}{\mathbf{k}_{i}^{\dagger}[\widehat{M}]_{F P}^{-1} \mathbf{k}_{i}} .
$$

This approach has been used in [3] by Conte et al. to derive a recursive algorithm for estimating the matrix $[M]$. This algorithm consists in computing the Fixed Point of $f$ using the sequence $\left([M]_{i}\right)_{i \geq 0}$ defined by:

$$
[M]_{i+1}=f\left([M]_{i}\right) .
$$


This study has been completed by the work of Pascal et al. [4], [5], which recently established the existence and the uniqueness, up to a scalar factor, of the Fixed Point estimator of the normalized covariance matrix, as well as the convergence of the recursive algorithm whatever the initialization. The algorithm can therefore be initialized with the identity matrix $[M]_{0}=\left[I_{m}\right]$.

It has also been shown in [6] and [3] that the recursive estimation scheme from Eq. 4 can be applied to derive an exact ML estimator of the normalized covariance matrix:

$$
[\widehat{M}]_{M L}=\frac{1}{N} \sum_{i=1}^{N} \frac{h_{m+1}\left(\mathbf{k}_{i}^{\dagger}[\widehat{M}]_{M L}^{-1} \mathbf{k}_{i}\right)}{h_{m}\left(\mathbf{k}_{i}^{\dagger}[\widehat{M}]_{M L}^{-1} \mathbf{k}_{i}\right)} \mathbf{k}_{i} \mathbf{k}_{i}^{\dagger}
$$

with

$$
h_{m}(q)=\int_{0}^{\infty} \tau^{p} \exp \left(\frac{q}{\tau}\right) p(\tau) d \tau .
$$

In Eq. 5. the exact ML estimator depends on the texture PDF through the SIRV density generating function $h_{m}(q)$. Chitour and Pascal have been recently demonstrated that Eq. 5 admits a unique solution and that its corresponding iterative algorithm converges to the Fixed Point solution for every admissible initial condition [7]. Pascal et al. have also demonstrated that normalized covariance ML estimator developed under the deterministic texture case (Eq. 3), yields also an approximate ML estimator under stochastic texture hypothesis [4], [5].

We propose to apply these results in estimating normalized coherency matrices for high resolution POLSAR data. The main advantage of this approach is that the local "scene heterogeneity" can be taken into account without any "a priori" hypothesis regarding the texture random variable $\tau$ (Eq.4 does not depend on $\tau$ ). The obtained Fixed Point is the approximate ML estimate under the stochastic $\tau$ assumption and the exact ML under deterministic $\tau$ assumption. Moreover, the normalized polarimetric coherency matrix estimated using the Fixed Point method is unbiased and asymptotically Gaussian distributed [4], [5].

Note also that the texture estimator from Eq. 2 can be directly linked to the total scattered power (span). By estimating the normalized coherency as the Fixed Point solution of Eq. 3. the derived estimate is independent of the total power and it contains polarimetric information only. Using this matrix, it is possible to compute the SIRV span ML estimator for unknown deterministic $\tau$ as:

$$
\widehat{P}_{P W F}=\mathbf{k}^{\dagger}[\widehat{M}]_{F P}^{-1} \mathbf{k} .
$$

Finally, it is possible to derive an estimate of the conventional polarimetric coherency matrix according to Eq. 1.

$$
[\widehat{T}]_{F P}=\frac{\widehat{P}_{P W F}}{m}[\widehat{M}]_{F P} .
$$

The proposed method is applied on airborne POLSAR images provided by the ONERA RAMSES system. The POLSAR data set was acquired over Toulouse, France (CNES headquarters) with a mean incidence angle of $50^{\circ}$. It represents a fully polarimetric (monostatic mode) X-band acquisition with a spatial resolution of approximately $50 \mathrm{~cm}$ in range and azimuth. The effectiveness of the Fixed Point estimator in compound Gaussian clutter can be observed in Fig. 1 . (a) with the color composition of the normalized coherency (Eq. 3) diagonal elements $[M]_{11}-[M]_{33}-[M]_{22}$. The selected scene is composed of both Gaussian (streets and fields) and non-Gaussian (urban) areas. Finally, the conventional polarimetric coherency matrix illustrated in Fig. 1.(b) is obtained according to Eq. 8

\section{GLRT SIMILARITY MEASURE}

Ground cover segmentation with POLSAR data is an important application. Generally, one has to find a similarity measure between the pixel covariance matrix $[M]$ and the class center $\left[M_{\omega}\right]$. We propose the following general binary hypothesis test for a given segment $\omega$ :

$$
\left\{\begin{array}{l}
H_{0}:[M]=\left[M_{\omega}\right] \\
H_{1}:[M] \neq\left[M_{\omega}\right]
\end{array}\right.
$$

According to the Neyman-Pearson Lemma, the LRT (Likelihood Ratio Test) provides the most powerful test:

$$
\Lambda=\frac{p_{m}\left(\mathbf{k}_{1}, \ldots, \mathbf{k}_{N} / H_{1}\right)}{p_{m}\left(\mathbf{k}_{1}, \ldots, \mathbf{k}_{N} / H_{0}\right)}
$$

In the case of the SIRV model, one can rewrite the hypothesis test as:

$$
\left\{\begin{array}{l}
H_{0}: \mathbf{k}=\sqrt{\tau} \mathbf{z}, \text { with } \mathbf{z} \sim \mathcal{N}(0,[M]) \\
H_{1}: \mathbf{k}=\sqrt{\tau} \mathbf{z}, \text { with } \mathbf{z} \sim \mathcal{N}\left(0,\left[M_{\omega}\right]\right)
\end{array}\right.
$$

where $\tau$ is the unknown deterministic texture.

For a given segment $\left[M_{\omega}\right]$, the LRT with respect to the texture $\tau$ and the normalized coherency matrix $[M]$ is given by:

$$
\Lambda_{S I R V}=\frac{\prod_{n=1}^{N} \frac{1}{\pi^{m} \tau_{n}^{m} \operatorname{det}\left\{\left[M_{\omega}\right]\right\}} \exp \left\{-\frac{\mathbf{k}_{n}^{\dagger}\left[M_{\omega}\right]^{-1} \mathbf{k}_{n}}{\tau_{n}}\right\}}{\prod_{n=1}^{N} \frac{1}{\pi^{m} \tau_{n}^{m} \operatorname{det}\{[M]\}} \exp \left\{-\frac{\mathbf{k}_{n}^{\dagger}[M]^{-1} \mathbf{k}_{n}}{\tau_{n}}\right\}} .
$$

Notice the likelihood function in Eq. 12 does not use the stochastic texture description as the $\operatorname{PDF} p(\tau)$ is supposed unknown in the SIRV model. Thus, the texture parameter $\tau$ can be considered either as a random variable with unknown PDF $p(\tau)$ or as an unknown deterministic parameter with PDF $p(\tau)=\delta\left(\tau-\tau_{n}\right)$ which characterizes yet a particular SIRV process. It can be shown that the ML estimation of the coherency matrix yields a good approximate ML estimate in the first case and the true ML estimate in the second case [6], 
[3] . The general PDF being unknown, it is therefore impossible to derive a texture independant closed-form expression for the Likelihood Ratio of the test given by Eq. 11. This procedure is here simplified, considering a particular SIRV process with a texture characterized by an unknown deterministic parameter. Consequently, each resolution cell is now associated with its own $p(\tau)=\delta\left(\tau-\tau_{n}\right)$, where $\tau_{n}$ are the unknown deterministic texture variables. This way the texture descriptor can be discarded for each pixel independently.

By taking the natural logarithm, one obtains:

$$
\begin{aligned}
\ln \left(\Lambda_{S I R V}\right) & =-N \ln \frac{\operatorname{det}\left\{\left[M_{\omega}\right]\right\}}{\operatorname{det}\{[M]\}}- \\
& -\sum_{n=1}^{N} \frac{\vec{k}_{n}^{\dagger}\left(\left[M_{\omega}\right]^{-1}-[M]^{-1}\right) \vec{k}_{n}}{\tau_{n}} .
\end{aligned}
$$

Now, since the $\tau_{n}$ 's and $[M]$ are unknown, they are replaced by their ML estimates from Eq. 2 and Eq. 3 . The resulting Generalized Likelihood Ratio Test $\Lambda_{S I R V}^{\prime}$ is given by:

$$
\begin{aligned}
\ln \left(\Lambda_{S I R V}^{\prime}\right) & =-N \ln \frac{\operatorname{det}\left\{\left[M_{\omega}\right]\right\}}{\operatorname{det}\left\{[\widehat{M}]_{F P}\right\}}- \\
& -m \sum_{n=1}^{N} \frac{\vec{k}_{n}^{\dagger}\left[M_{\omega}\right]^{-1} \vec{k}_{n}}{\vec{k}_{n}^{\dagger}\left[\widehat{M}_{F P}^{-1} \vec{k}_{n}\right.}+N m .
\end{aligned}
$$

Maximizing the GLRT over all segments is equivalent to minimizing the following similarity measure:

$$
D_{S I R V}=\ln \frac{\operatorname{det}\left\{\left[M_{\omega}\right]\right\}}{\operatorname{det}\left\{[\widehat{M}]_{F P}\right\}}+\frac{m}{N} \sum_{n=1}^{N} \frac{\vec{k}_{n}^{\dagger}\left[M_{\omega}\right]^{-1} \vec{k}_{n}}{\vec{k}_{n}^{\dagger}[\widehat{M}]_{F P}^{-1} \vec{k}_{n}}
$$

\section{OPTIMAL GLRT SEGMENTATION}

We consider the POLSAR segmentation as the following multiple composite hypothesis test:

$$
\left\{\begin{array}{l}
H_{0}: \mathbf{k}=\sqrt{\tau} \mathbf{z}, \text { with } \mathbf{z} \sim \mathcal{N}(0,[M]) \\
H_{1}: \mathbf{k}=\sqrt{\tau} \mathbf{z}, \text { with } \mathbf{z} \sim \mathcal{N}\left(0,[M]_{1}\right) \\
\ldots \\
H_{i}: \mathbf{k}=\sqrt{\tau} \mathbf{z}, \text { with } \mathbf{z} \sim \mathcal{N}\left(0,[M]_{i}\right) \\
\ldots \\
H_{\mathcal{C}}: \mathbf{k}=\sqrt{\tau} \mathbf{z}, \text { with } \mathbf{z} \sim \mathcal{N}\left(0,[M]_{\mathcal{C}}\right)
\end{array}\right.
$$

where $\tau$ is the unknown deterministic texture, $\mathcal{C}$ is the number of segments and $[M]$ is the normalized covariance matrix of the observed independent identically distributed $N$ secondary data. Notice that the segmentation in $\mathcal{C}$ segments is equivalent to testing $\mathcal{C}+1$ hypotheses.

In this paper we suppose that the multiple hypotheses from Eq. 16 are not nested, i.e. there is a unique normalized covariance matrix characterizing each class. This implies that each class is described by a different SIRV, and hence the extended GLRT can be applied.
In multiple composite hypothesis test, the likelihood ratios are sufficient for optimal segmentation problem. The use of likelihood ratios referenced to a "dummy" hypothesis has been introduced in [8]. In consequence, the secondary data observed at instance $t+1$ are assigned to the segment $\omega$, which maximizes the extended GLRT over all classes characterized by their normalized covariance matrices estimated at instance $t$ :

$$
\omega=\arg \max _{1 \leq i \leq \mathcal{C}} \frac{p_{m}\left(\mathbf{k}_{1}, \ldots, \mathbf{k}_{N} / H_{i}\right)}{p_{m}\left(\mathbf{k}_{1}, \ldots, \mathbf{k}_{N} / H_{0}\right)}=\arg \max _{1 \leq i \leq \mathcal{C}} \Lambda_{i}
$$

where $p_{m}\left(\mathbf{k}_{1}, \ldots, \mathbf{k}_{N} / H_{0}\right)$ is the PDF under $H_{0}$ and $p_{m}\left(\mathbf{k}_{1}, \ldots, \mathbf{k}_{N} / H_{\omega}\right)$ is the PDF under $H_{1}$ from the setup of Eq.11] in Sect. 3

Using the results obtained in Sect. 3, the condition from Eq. 17 is equivalent to minimizing:

$$
\begin{aligned}
\omega & =\arg \max _{1 \leq i \leq \mathcal{C}}\left\{\ln \frac{\operatorname{det}\left\{\left[\widehat{M}_{i}\right]_{F P}\right\}}{\operatorname{det}\left\{\left[\widehat{M}_{F P}\right\}\right.}+\right. \\
& \left.+\frac{m}{N} \sum_{n=1}^{N} \frac{\vec{k}_{n}^{\dagger}\left[\widehat{M}_{i}\right]_{F P}^{-1} \vec{k}_{n}}{\vec{k}_{n}^{\dagger}[\widehat{M}]_{F P}^{-1} \vec{k}_{n}}\right\},
\end{aligned}
$$

where $\left[\widehat{M}_{i}\right]_{F P}$ at the instance $t$ is estimated from the segment $i$ at instance $t-1$ using the Fixed Point estimator. The segmentation algorithm is given in Algorithm 1.

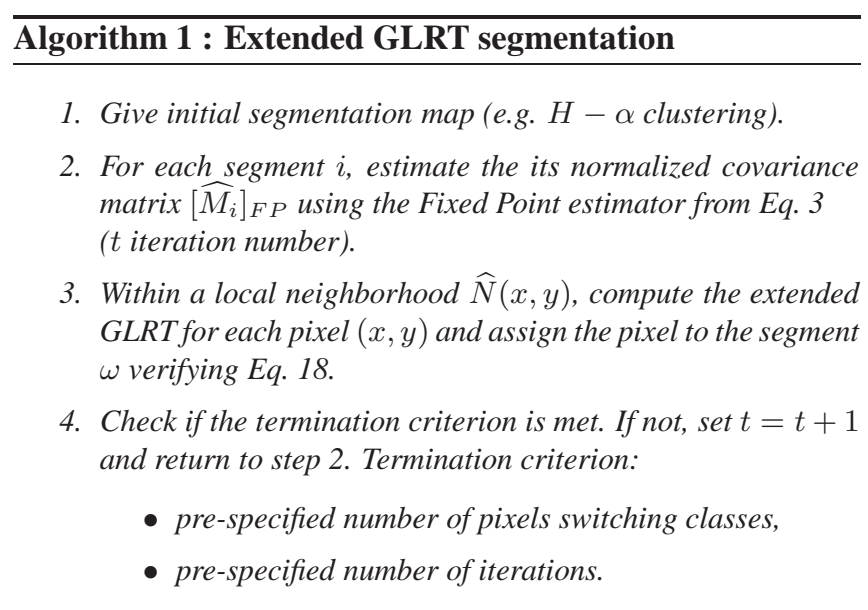

Fig. 1-(c) illustrates the POLSAR unsupervised segmentation results using the normalized coherency matrix descriptor. One key issue to be discussed is weather the normalized coherency matrix (NC) and the span should be aggregated in the final estimation step or not. Most of the existing processing chains use the conventional coherency matrix for representing POLSAR data for unsupervised land cover classification and for target detection applications. Due to the SIRV model identification problem, the complete description of the POLSAR data set is achieved by estimating the span and the normalized coherency independently. The joint analysis of 
the span and the normalized coherency presents several advantages with respect to the coherency matrix descriptor: separation between the total received power and the polarimetric information, estimation of the NC independently of the span and the existence of the SIRV distance measure for unsupervised ML segmentation of normalized coherencies. However, the span-NC description of POLSAR images raises new problems which still remain under investigation. Although all statistical requirements employed for unsupervised segmentation are met, the polarimetric information is quite difficult to extract. As it can be noticed in Fig. 1-(c), the polarimetric signatures are strongly mixed and the class boundaries are smoothed within high resolution POLSAR images (even for highly heterogeneous target areas).

\section{CONCLUSION}

This paper presented a new estimation scheme for deriving normalized coherency matrices and the resulting estimated span with high resolution POLSAR images. The heterogeneous clutter in POLSAR data was described by the SIRV model. The Fixed Point estimation is independent on the span PDF and represents an approximate ML estimator for a large class of stochastic processes obeying the SIRV model. For SIRV clutter, a new ML distance measure was also introduced for optimal unsupervised POLSAR segmentation.

\section{REFERENCES}

[1] M. S. Greco and F. Gini, "Statistical analysis of high-resolution SAR ground clutter data," IEEE Transactions on Geoscience and Remote Sensing, vol. 45, no. 3, pp. 566-575, 2007.

[2] K. Yao, "A representation theorem and its applications to sphericallyinvariant random processes," IEEE Transactions on Information Theory, vol. 19, no. 5, pp. 600-608, 1973.

[3] E. Conte, A. DeMaio, and G. Ricci, "Recursive estimation of the covariance matrix of a compound-Gaussian process and its application to adaptive CFAR detection," IEEE Transactions on Image Processing, vol. 50, no. 8, pp. 1908-1915, 2002

[4] F. Pascal, Y. Chitour, J. P. Ovarlez, P. Forster, and P. Larzabal, "Covariance structure maximum-likelihood estimates in compound Gaussian noise: existence and algorithm analysis," IEEE Transactions on Signal Processing, vol. 56, no. 1, pp. 34-48, 2008.

[5] F. Pascal, P. Forster, J. P. Ovarlez, and P. Larzabal, "Performance analysis of covariance matrix estimates in impulsive noise," IEEE Transactions on Signal Processing, vol. 56, no. 6, pp. 2206-2216, 2008.

[6] F. Gini and M. V. Greco, "Covariance matrix estimation for CFAR detection in correlated heavy tailed clutter," Signal Processing, vol. 82, no. 12, pp. 1847-1859, 2002.

[7] Y. Chitour and F. Pascal, "Exact maximum likelihood estimates for SIRV covariance matrix: existence and algorithm analysis," IEEE Transactions on Signal Processing, vol. 56, no. 10, pp. 4563-4573, 2008.

[8] H. L. VanTrees, Detection, estimation, and modulation theory, JohnWiley\&Sons, New York, USA, 1968.

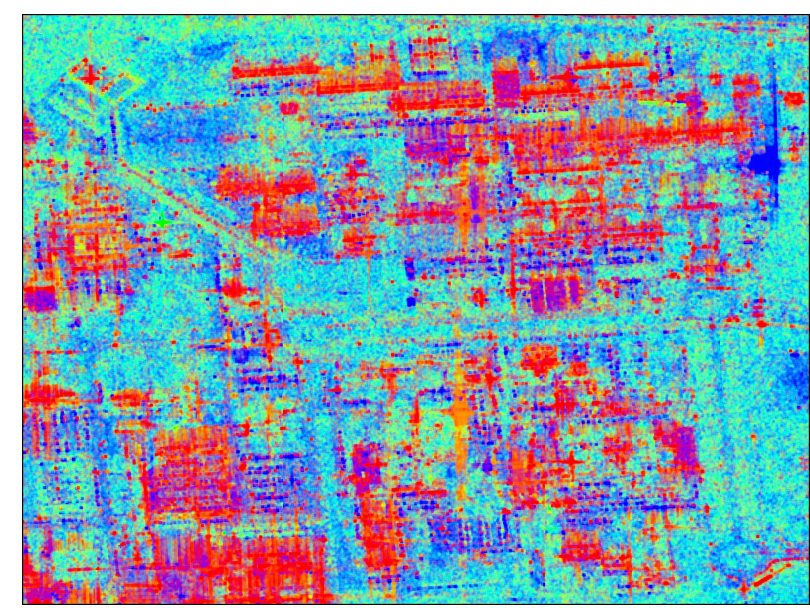

(a)

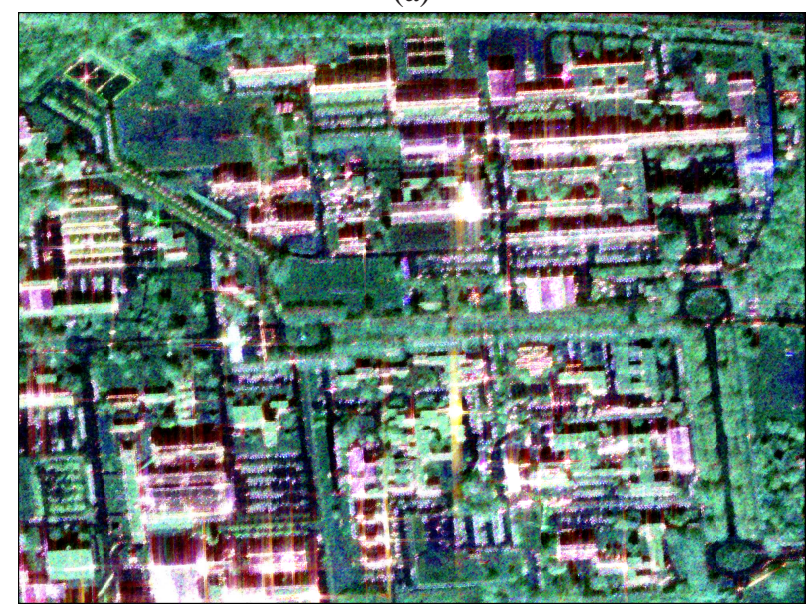

(b)

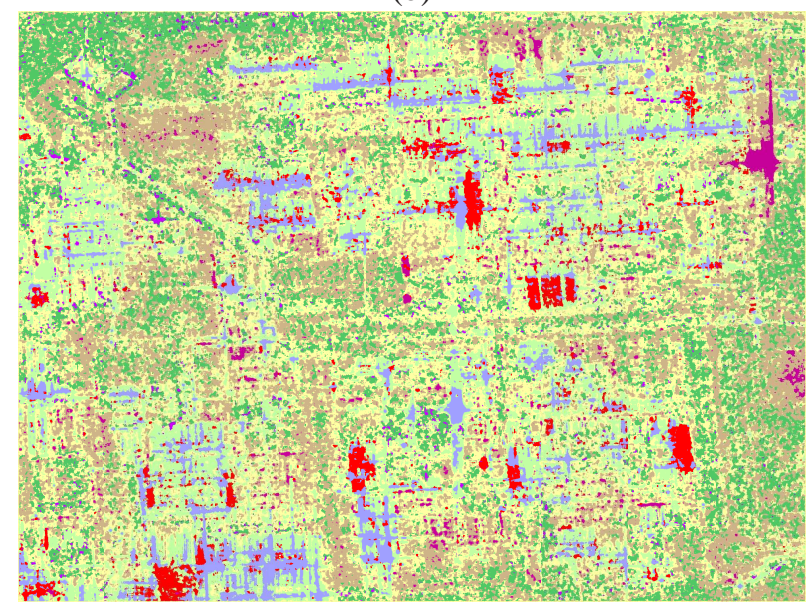

(c)

Fig. 1. RAMSES data $(1500 \times 2000$ pixels $)$ : (a) color composition of the normalized coherency (Eq. 3) diagonal elements $[M]_{11}$ $[M]_{33}-[M]_{22}$, (b) color composition of the coherency (Eq. [8) diagonal elements $[T]_{11-}-[T]_{33-}-[T]_{22}$, (c) unsupervised ML segmentation of the normalized coherency (8-segment $H-\alpha$ initialization). 